\title{
Visualization, Interactive Handling and Simulation of Molecules in Commodity Augmented Reality in Web Browsers Using moleculARweb's Virtual Modeling Kits
}

\author{
Fabio Cortés Rodríguez, Lucien F. Krapp, Matteo Dal Peraro, and Luciano A. Abriata*
}

\begin{abstract}
ARweb (https://molecularweb.epfl.ch) began as a website for education and outreach in chemistry and structural biology through augmented reality (AR) content that runs in the web browsers of regular devices like smartphones, tablets, and computers. Here we present two evolutions of moleculARweb's Virtual Modeling Kits (VMK), tools where users can build and view molecules, and explore their mechanics, in 3D AR by handling the molecules in full 3D with custom-printed cube markers (VMK 2.0) or by moving around a simulated scene with mouse or touch gestures (VMK 3.0). Upon simulation the molecules experience visually realistic torsions, clashes, and hydrogen-bonding interactions that the user can manually switch on and off to explore their effects. Moreover, by manually tuning a fictitious temperature the users can accelerate conformational transitions or 'freeze' specific conformations for careful inspection in 3D. Even some phase transitions and separations can be simulated. We showcase here these and other features of the new VMKs connecting them to possible specific applications to teaching and self-learning of concepts from general, organic, biological, and physical chemistry; and in assisting with small tasks in molecular modelling for research. Last, in a short discussion section we overview what future developments are needed for the 'dream tool' for the future of chemistry education and work.
\end{abstract}

Keywords: Augmented reality · Chemistry education · Molecular graphics · Molecular modelling · Molecular simulations - Web programming - WebXR

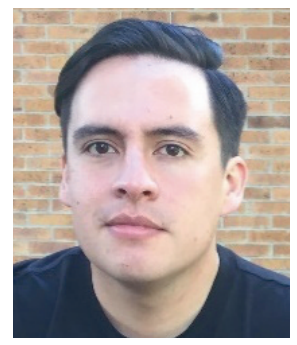

Fabio Cortés is a software engineer, passionate about augmented and virtual reality and exploring the intersection between art and technology. Currently he works as WebXR engineer at the Laboratory for Biomolecular Modeling at EPFL creating AR/VR tools for teaching, learning, and working on chemistry and structural biology.

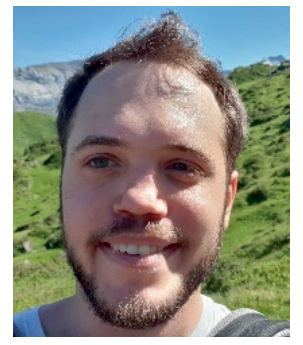

Lucien F. Krapp is a PhD student in physics at the Laboratory for Biomolecular Modeling in the School of Life Sciences at EPFL. He has a MSc in Physics with a minor in Computational Science and Engineering from EPFL. Currently, he is involved in method development at the Laboratory for Biomolecular Modeling applying machine learning to structural biology and biophysics. He has experience in data analysis, machine learning, and deep learning.

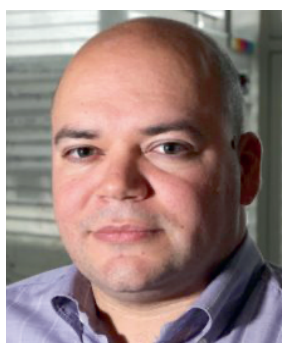

Prof. Dr. Matteo Dal Peraro heads the Laboratory for Biomolecular Modeling at EPFL since 2007. He graduated in Physics at the University of Padua in 2000 and obtained his $\mathrm{PhD}$ in Biophysics at the International School for Advanced Studies (SISSA, Trieste) in 2004. The research of his group, within EPFL's Interfaculty Institute of Bioengineering, focuses on the multiscale integrative modeling of large macromolecular systems, molecular simulations, and engineering and applications of bioinspired nanopores.

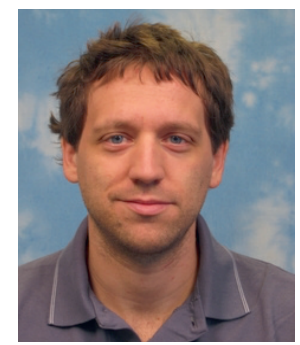

Dr. Luciano A. Abriata is a scientist at the Laboratory for Biomolecular Modeling and at the Protein Structure Core Facility, both in EPFL's School of Life Sciences. Biotechnologist and PhD in Chemistry with experience in the wet and dry labs, he currently works on various structural biology/ biophysics projects and guides the group's developments of novel human-computer interfaces for teaching, learning and working in chemistry and structural biology. 


\section{Introduction}

Launched during 2019-2020, moleculARweb (https://molecularweb.epfl.ch/) is a website for chemistry and structural biology education through augmented reality (AR) that works on consumer devices like smartphones, tablets and laptops. ${ }^{[1]}$ The website provides various web apps whereby students and educators can manipulate virtual molecules and other chemistry-related entities such as electronic orbitals, density maps, etc. in 3D space by presenting paper-printed fiducial markers to the webcams of their devices (in certain static visualizations, especially for structural biology topics, the virtual objects can also be anchored to surfaces and then the user can move freely around them; these apps are not treated in this paper but are described in ref. [2]). Each app inside moleculARweb is delivered as a regular webpage, so users do not need to install any programs to use them. They simply have to access the web page with the activity of interest, enable the webcam, and present it the fiducial markers, onto which the app will display the relevant objects. The website quickly got thousands of users (data constantly monitored at https://clustrmaps.com/site/1ar2f), many of them accessing during the COVID-19 pandemic from their homes thanks to the simplicity of the system. Online and in-person surveys showed positive effects on learning, strong engagement by students, and high success rates in making the AR activities work, ${ }^{[1]}$ the latter likely due to the entirely web-based nature of the tools which facilitates deployment as noticed by multiple works. ${ }^{[3,4]}$ More globally, beyond our specific resource, augmented reality has been widely used to build all kinds of educational tools to assist chemistry teaching and learning, from small molecule display to reaction mechanisms, structural biology, and even 'virtual' lab sessions. ${ }^{[4-7]}$

Since the release of moleculARweb teachers have been increasingly requesting more material and features, especially for its most accessed app: the Virtual Modeling Kit (VMK), a tool to display in AR any molecule loaded from its atomic coordinates in PDB format, attached to a 3D cube marker which facilitates handling (i.e. translation and rotation) of the molecule in real 3D space. The main requests made by the teachers concerned the availability of a larger selection of molecules, the possibility of loading multiple molecules and that they could interact with each other, plus some case-specific features. Here we present two separate evolutions of the early VMK (still supported in legacy mode) that address these requests and incorporate further features, some of which may prove useful even for basic tasks in chemical research work. These new apps are called VMK 2.0 and VMK 3.0, both available under the module called Molecular modeling kit at https://molecularweb.epfl.ch. The VMK 2.0 supports loading molecules on two separate fiducial 3D cube markers, enabling their independent manipulation in space. The VMK 3.0 does not work with fiducial markers; rather, the molecules float inside a (transparent) simulation box as they are loaded. In both new VMKs the molecules are subject to rudimentary physics based on a rigid-body model that enables visually realistic mechanics, including diffusion, evolution of internal degrees of freedom, and non-covalent interactions between molecules. Both new VMKs support loading molecules in various ways, even drawing any arbitrary molecule by hand. And like the rest of the moleculARweb website, both new VMKs work smoothly on laptops, smartphones and tablets. Here we describe in detail all these features plus other smaller gadgets and options, connecting to specific case uses for education and small applications in research, and advancing possible routes for future developments.

\section{Programming and Theoretical Basis}

Like the rest of moleculARweb's apps, the VMKs rely on client-side web programming technologies, combining HTML, CSS and WebGL for visual presentation and JavaScript for interactivity. ${ }^{[8-11]}$ All code is directly accessible on the author FCR's GitHub account (https://github.com/fcor/molecular-mirror-app) and can also be viewed right in-place (Ctrl+U in most browsers).

The VMKs use Three.js as the 3D graphics library, from which atoms and bonds are respectively rendered using sphere and cylinder primitives - in contrast to other apps in moleculARweb and in other software where the objects are pre-made 3D models, and hence lack interactivity. The VMK 2.0 uses the Ar.js library to detect and track the 3D cube markers, of which marker 1 is the same used by the early VMK and marker 2 was created for the new app. In each render cycle the molecules are aligned to their starting conformation, centered, and rotated onto the orientation of the corresponding 3D cube marker. When a cube is not detected (e.g. webcam glitches, occlusion, or simply not in the field of view) the molecule is fit and positioned to the last known location and orientation. The VMK 3.0 lacks markers, because the molecules float inside an invisible box. The user can move around this simulation box with touch gestures on smartphones and tablets or with the mouse moves and scrolls in laptops and desktop computers.

To simulate molecular mechanics, the VMKs use the Cannon.js engine for rigid body physics through efficient Verlet-like stepper integrations under constraints ${ }^{[12]}$ designed to achieve visually reasonable dynamics. Briefly, atoms are described as hard Cannon spheres of radii proportional to atomic radii; pairs of atoms within bonding distance are kept at a fixed distance with a strong Cannon constraint, pairs of atoms bonded to the same atom are constrained to the starting distances to maintain angles, and pairs of atoms bonded to pairs of bonded $\mathrm{sp}^{2}$ carbons are constrained to hamper rotations around double $\mathrm{C}-\mathrm{C}$ bonds, which naturally propagates to other elements such as aromatic rings, conjugated double bonds, peptide bonds, etc. While atoms always move under the imposed physics, at each render cycle their velocities are randomly changed and rescaled to match an expected 'temperature'. The user increases or decreases this 'temperature' at will through the + and - buttons of the VMK apps, but the setting remains hidden because although higher values result in faster and more ample motions as expected, the exact numbers lack any physical realism. In fact this 'temperature' only serves the purpose of controlling the extent of molecular dynamics. In the VMK 3.0 the atomic fluctuations naturally couple into translational and rotational diffusion plus internal molecular motions; instead, in the VMK 2.0 the molecules are aligned to their starting conformation at each iteration thus effectively removing translational and rotational diffusion and leaving only the internal degrees of freedom active, useful to highlight conformational changes.

For molecule building and retrieval the VMKs use the JSME applet ${ }^{[13]}$ and CACTUS's Chemical Identifier Resolver at the U.S. National Cancer Institute. ${ }^{[14]}$

\section{Virtual Modeling Kit 2.0: Handling Molecules in 3D Space in Augmented Reality}

The VMK 2.0 allows users to load one molecular system (each system can consist in one or multiple molecules at once) onto each of two 3D cube markers that they can then move around independently. Virtually any molecule can be copy-pasted into the text box in the PDB-like format of the examples, drawn manually with the embedded JSME app, retrieved from CACTUS NCI, ${ }^{[14]}$ 
or chosen from a pre-made list that includes a selection of molecules requested by teachers spanning from small molecules of interest in general and organic chemistry to small biological molecules and small pieces of large systems such as proteins, DNA, ice formations, inorganic crystals, and carbon allotropes. We note that these all serve as quick example molecules, but any other imaginable molecule made up of any element of the periodic table is supported, copy-pasted into the PDB code box, or drawn in the JSME app, or retrieved from CACTUS. In practice, low-end laptops and high-end smartphones support up to 200-500 total atoms, which we consider more than enough given that these apps are devised to work with small molecules only, while moleculARweb offers other tools more suitable for larger (bio)molecules and assemblies. ${ }^{[1,2]}$

On clicking 'Start AR' in the VMK 2.0 the program parses the molecular system as described under section 2 and displays it anchored to the selected cube marker. The cube markers can be printed and folded from pages 3 and 4 of the PDF linked in Table 1. At least two teachers (Prof. Rodolfo Rasia at IBR, Argentina; and Dr. Jonathan Ferooz at ATGstart, Belgium) have produced 3D-printable versions of these cubes (links in Table 1). More details on how to use the VMK 2.0 are given in the extended information online linked in Table 1, which also includes links to videos on how to print, prepare and use the markers (essentially the same as described in available in the original moleculARweb article $^{[1]}$ but extended to two cube markers).

The VMK 2.0 features a set of controls specific to each marker that govern whether the molecules are displayed as small spheres and sticks or as larger van der Waals spheres and whether each molecule is to be inverted or not. The former option allows switching between a view that highlights bonding and dynamics (sticks) and a view that more accurately represents the volume actually taken up by a molecule (spheres) (Fig. 1A). The invert option serves to explore chiral centers; for example, the user could load the same molecule on both markers and then invert only one to check whether the two molecules indeed correspond to the same or rather chirality makes them different (Fig. 1B). A global option within the same panel controls whether clashes and hydrogen bonds between atoms of molecules held must be shown or not, and whether attractive hydrogen bonding forces should be applied between molecules of two markers or not (Fig. 1C). This last point in turn relates to the simulation of mechanics, which is controlled by a separate panel symbolized with a thermometer. Here, the user can increase or decrease an artificial temperature that induces atomic motions, which are coupled throughout the structure of a molecule based on a set of connectivity rules defined by its starting conformation (see section 2). Interesting applications of this to an educational setting include comparing rigid vs. flexible features of molecules and exploring the conformational landscapes of simple molecules. One can, for example, compare the rigidity of double vs. simple bonds (Fig. 1D) or aromatic vs. aliphatic cycles; or explore rotations around dihedral angles; or exchange between chair and boat conformations in 6-membered rings. As exemplified in Fig. 1E, the latter can be made visually clearer by placing regular $\mathrm{H}$ and dummy $\mathrm{H}$ atoms ( $\mathrm{HX}$ ) of different colors in axial and equatorial positions (similar dummy atoms exist for $\mathrm{C}, \mathrm{N}$ and $\mathrm{O}$ too).

In research, the VMK 2.0 could be useful for building and retrieving molecules from CACTUS, possibly further copied into other programs as prepared or after simulation by clicking on 'Copy current coordinates' (Fig. 1F); also simply for visualizing molecules on any device and with no usage barriers, especially for comparing pairs of very similar molecules that differ in a few groups or just in chirality at certain centers -an operation that regular computer graphics programs just do not enable because human inputs are restricted to single molecules at a time (Fig. 1G).

\section{Virtual Modeling Kit 3.0: Simulation Boxes in Augmented Reality}

The VMK 3.0 accepts the same kinds of molecules as the VMK 2.0, and they can be inputted in the same four ways, but in the former the molecules are not anchored to any marker, and

Table 1. List of associated online resources and extended material.

\section{Resource}

Video on preparing the markers

Video on using the cube markers in VMK 2.0

PDF to print 3D markers

User-contributed files for 3D-printing the cube markers

Practical introduction to the whole molecularweb, VMKs included

Tutorial on all four ways to load molecules in the VMKs and how to view them

General tutorial on web-based AR for chemistry and biology education using moleculARweb and Google-based resources

\section{URL}

https://www.youtube.com/watch?v=Fo0WpTmUJ4s

https://youtu.be/GuYMqG8kce8?t=20

Pages 3 and 4 of https://molecularweb.epfl.ch/assets/markers/allmarkers.pdf

https://www.thingiverse.com/thing:4881962 or https://www.thingiverse.com/thing:4922818

https://lucianosphere.medium.com/molecularweb-a-brief-practical-introduction-5738771b224d

https://lucianosphere.medium.com/how-to-load-any-molecule-for-display-in-augmented-realityon-molecularweb-5da0af4b64b2

https://medium.com/geekculture/chemistry-and-biology-education-using-commodity-web-augmented-reality-in-any-device-9fa9fdc4ab35 
A

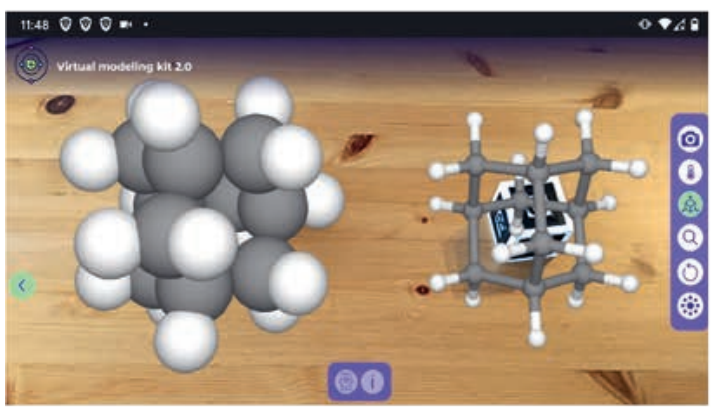

B
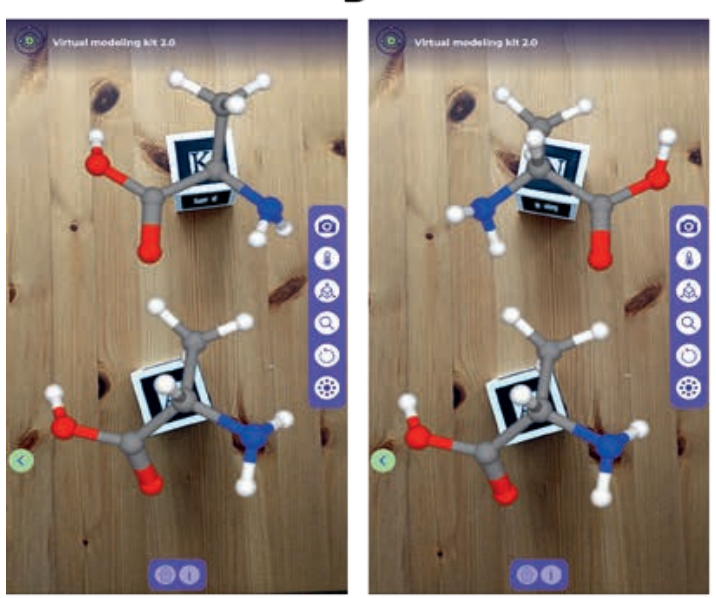

C

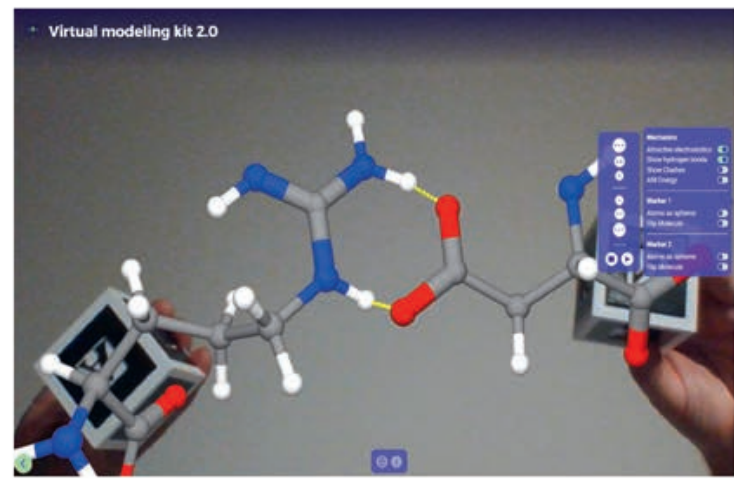

D

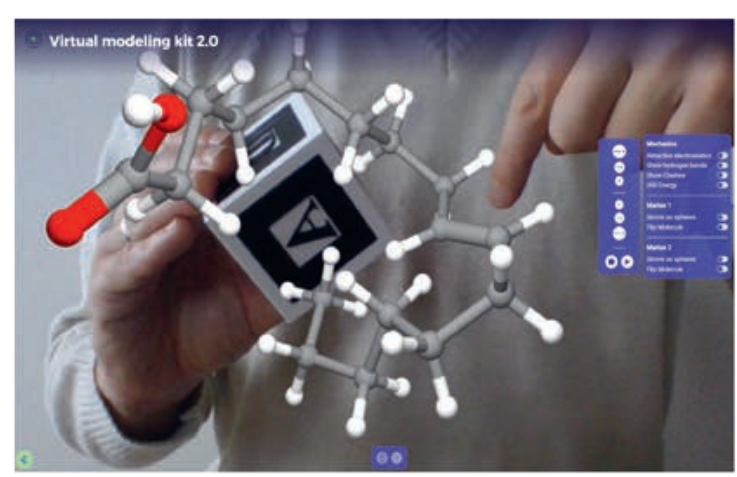

E
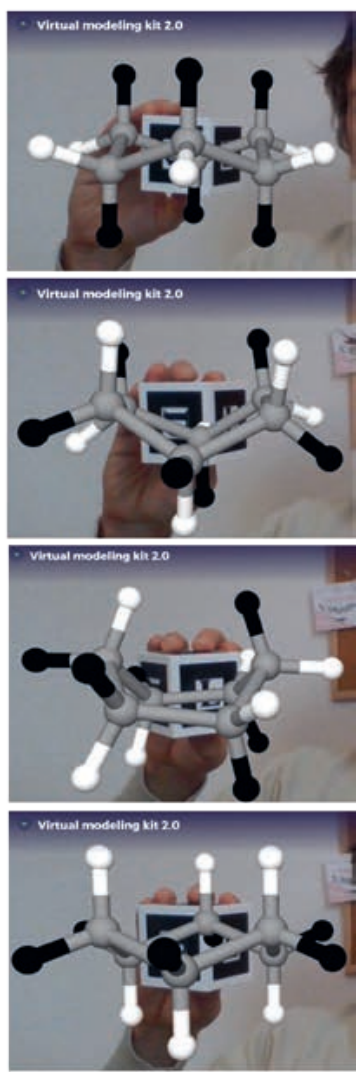

F

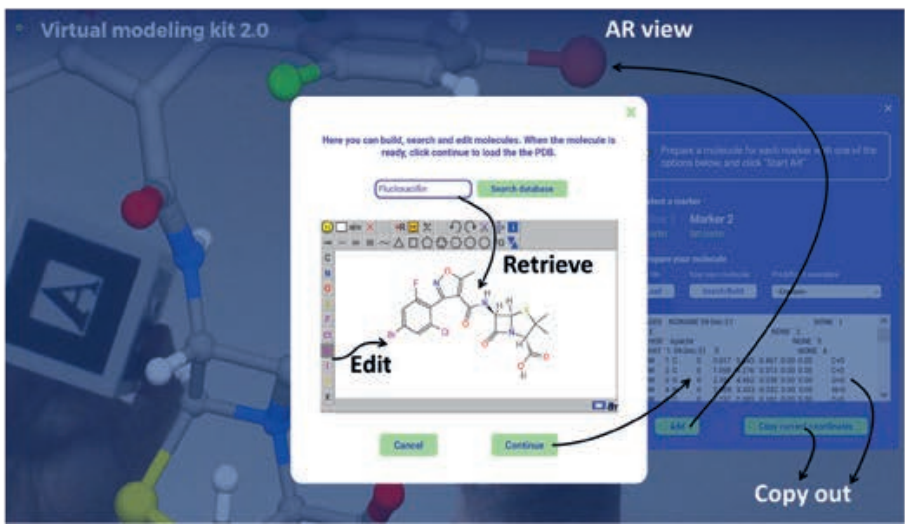

G

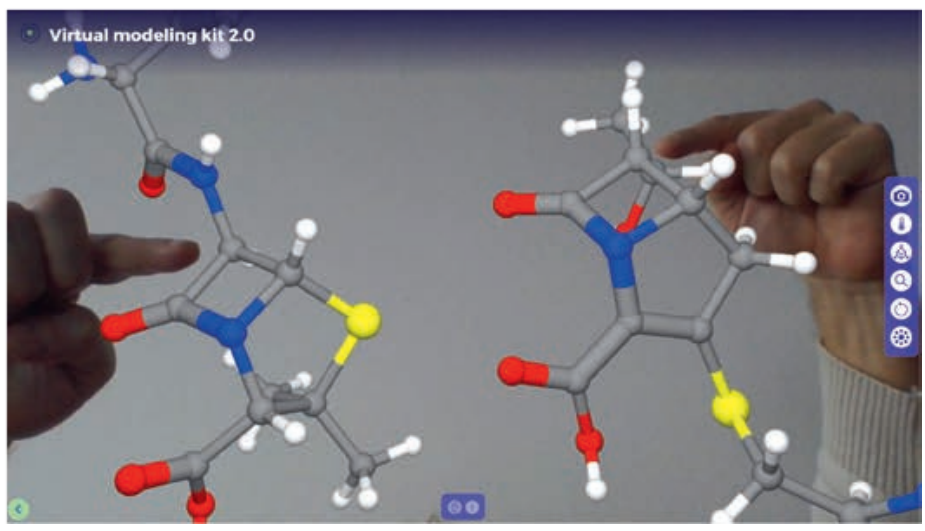

Fig. 1. Example applications of the VMK 2.0. (A) In pass-through mode on a smartphone in landscape orientation, comparing adamantane as sticks to better understand the spatial connectivity of its $C$ atoms and in spacefill view to better grasp its total volume. (B) In pass-through mode on a smartphone in portrait, L-alanine was loaded onto the two cubes and inverted in the top cube to get R-alanine. By rotating the cubes (right: the top cube is rotated by $180^{\circ}$ to match the orientation of the $\mathrm{H}$ and methyl groups in both molecules) it becomes obvious that there is no way to superimpose the molecules around the central $\mathrm{C}$ atom, in contrast to, for example, glycine, a non-chiral amino acid. (C) On a computer in selfie mode, an arginine amino acid hydrogen-bonded through its guanidinium group to a deprotonated aspartate. (D) On a laptop in selfie mode, upon simulation of palmytoleic acid at high temperature the planarity around the double $\mathrm{C}=\mathrm{C}$ bond (pointed at) and at the carboxylic group are retained, while the simple C-C bonds freely explore the dihedral space. (E) A cyclohexane molecule whose axial Hs were replaced by a dummy XH atom which is parametrized like $\mathrm{H}$ but colored black. Upon simulation by increasing temperature, the molecule exchanges between alternate chair conformations where axial and equatorial Hs have swapped, through boat conformations. (F) Retrieving a molecule, here Flucloxacillin, editing it to add a Br atom and then viewing the molecule in-app and exporting the resulting PDB (or the result of its motions upon simulation) for use in external programs. (G) Comparing the canonical $\beta$-lactam antibiotic ampicillin (right) with the $\beta$-lactamase-resistant imipenem (left). After having oriented the molecules by moving the markers, the user points at the main difference between the two molecules, which other than the different substituents consists in the opposite chirality of the $\mathrm{C}$ atom alpha to the amide carbon of the $\beta$-lactam ring.

rather placed inside a simulation box where they are subject to the physical constraints imposed by the app controlled by the user. In the VMK 3.0 the user moves around the scene through the orbit controls made available by the device; for example mouse moves and scrolls on computers or touch gestures in smartphones and tablets. Like in the VMK 2.0, inter-atomic constraints are computed when the molecule is added and then the temperature, clashes and hydrogen bonding forces (controlled by the user) result in the simulated mechanics. Since molecules are not constantly realigned like in the VMK 2.0, atomic fluctuations 
eventually develop not only into internal dynamics but also into rotational and translational diffusion. Additionally, the VMK 3.0 allows action of an artificial gravity that pulls molecules down towards a virtual floor represented as a grid (when the gravity is on).

Compared with the VMK 2.0, the VMK 3.0 allows easier handling of molecules that are too big to be moved around with markers, but most importantly, it allows users to do 'experiments' unapproachable with the VMK 2.0. Among pedagogically interesting examples, one can explore the tradeoffs between attractive energetics (enthalpy-like) and disorder fostered by kinetic energy (entropy-like) and thus simulate how (in)miscibility of different molecules emerges from differences in polarity, demonstrate phase transitions, etc. Fig. 2A shows, for example, a piece of ice as setup from starting coordinates, then energy-minimized by activating hydrogen bonding without temperature, and then how it 'melts' when temperature is lightly increased, forming a 'droplet' that floats (note that the encoded physics are not good enough to return to crystalline ice when the temperature is dropped, but also take into account that freezing is extremely difficult to be observed, let alone quantitatively reproduced, even with high-end atomistic simulations ${ }^{[15]}$ ). In Fig. 2B a bulk of liquid-like water molecules sinks gravitationally to the bottom of the simulation box; then the temperature is increased until the water 'boils', and even before boiling one can see water molecules leaving the solution, i.e. the basis for vapor pressure. Fig. $2 \mathrm{C}$ presents a mixture of water and butane molecules that do not phase-separate because hydrogen bonding is inactive; when this attractive force is switched on, over time water molecules interact with each other excluding the butane molecules and thus forming two separate phases.

\section{A}
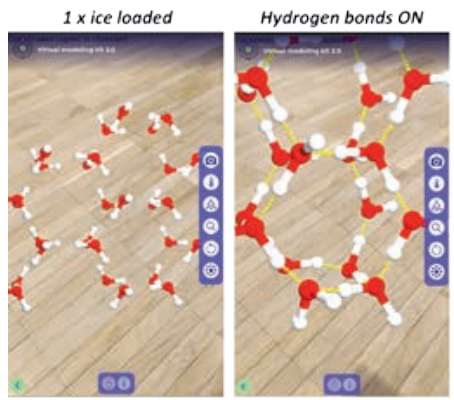

C

1 minute simulation of water + butane molecules, hydrogen bonding OFF

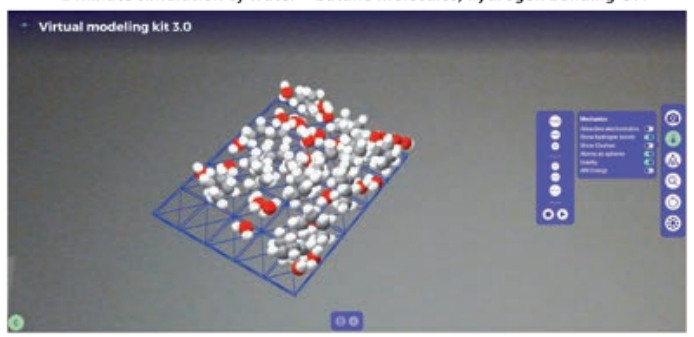

$\sim 1$ minute extension with hydrogen bonding $O N$

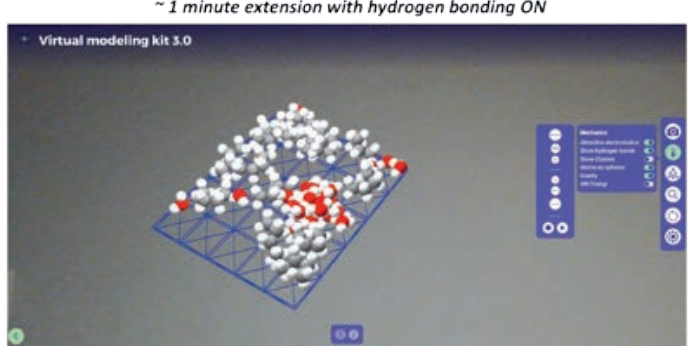

For research, just like the VMK 2.0, the VMK 3.0 serves for easily building and retrieving molecules from CACTUS, to visualize molecules on any device anywhere in the world with an internet connection and with no entry barriers (with the advantage in the VMK 3.0 that it requires no markers), and possibly to extract the starting or the simulated system out of the app in PDB format to be used in other applications by clicking on 'Copy current coordinates'. Compared to the VMK 2.0, the VMK 3.0 hardly serves to compare pairs of molecules or set them in specific locations and orientations, because they cannot be manually driven in space. However, the VMK 3.0 serves, for example, to set up systems with random molecular positions and orientations much like programs specifically dedicated to this such as Packmol[16] (example in Fig. 2D).

\section{Discussion}

We have briefly presented the new VMKs of our moleculARweb site, stressing their main functionalities. Additional features available in both VMKs include swapping cameras (useful to change between selfie and passthrough modes in devices like smartphones or tablets, as exemplified in the figures), zooming options, restart buttons (softer alternatives to webpage refresh), and in-line language choice (this feature does not work for molecule names). More detailed tutorials in the form of videos and blog entries are presented in Table 1 with direct links.

Online analytics, surveys, personal communications from students and especially from teachers disclosed extensive student engagement and applicability of the early and new versions of the VMK in classrooms. ${ }^{[1,17]}$ From this

B

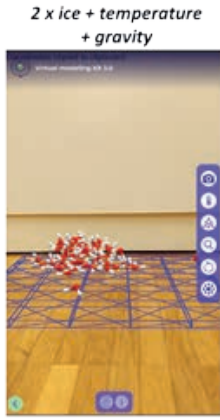

++ temperature

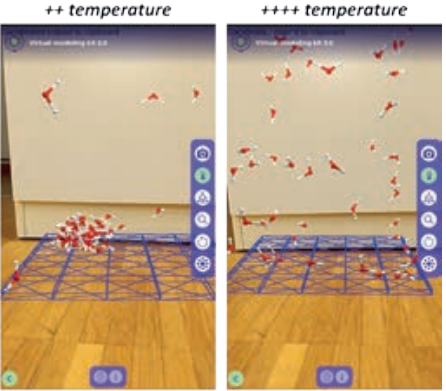

D
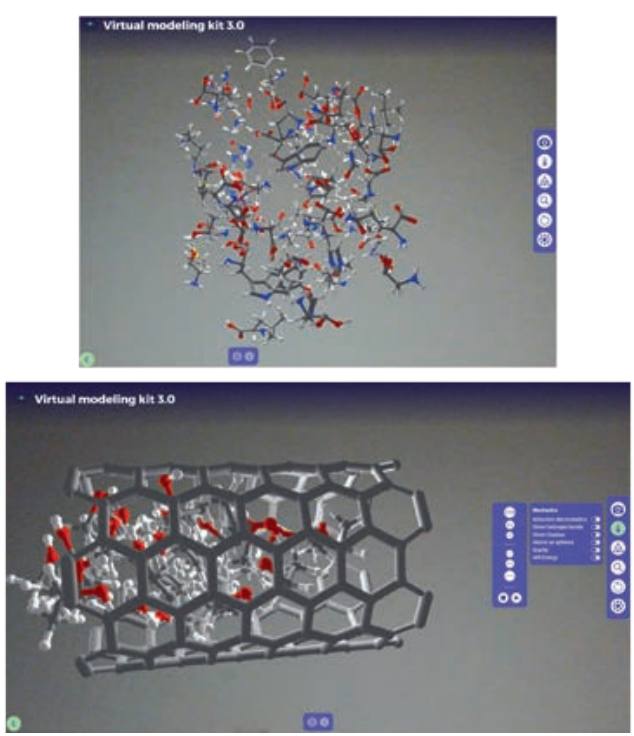

Fig. 2. Example applications of the VMK 3.0. (A) In passthrough mode on a smartphone in portrait orientation, a piece of ice is loaded, then minimized by activating hydrogen bonding, and melted by increasing temperature. (B) In pass-through mode on a smartphone in portrait orientation, a drop of water molecules lying at the bottom of the simulation box is slowly heated up until it becomes fully gaseous. (C) In pass-through mode on a tablet, a number of water and butane molecules are mixed and simulated first without and then with hydrogen bonding forces. These forces bring the water molecules together resulting in the expected phase separation. (D) Building systems for subsequent molecular simulation: a mixture of amino acids (2 of each) loaded in the VMK 3.0 and then dispersed through simulation (top); and a set of ethanol molecules placed inside a carbon nanotube. 
feedback plus our own use of the tools and considering the state of the art of the two main fields around the VMK, i.e. molecular simulations/modeling/informatics and VR/AR, we expect two major developments required to build the next generation of tools for education and work in chemistry.

\subsection{Immersivity}

The systems presented here have the advantage of being essentially cost-free and highly deployable compared to high-end $\mathrm{AR}$ and virtual reality (VR) alternatives, while improving over standard molecular graphics tools regarding some functionalities. As exemplified throughout the figures, they enable independent simultaneous control of two molecules (VMK 2.0) and the use of body limbs to point at multiple molecular features at the same time (both VMKs). However, the AR methodology used by our tools is limited regarding the level of molecule control and depth of $3 \mathrm{D}$ view. Towards better $3 \mathrm{D}$ views and especially more natural ways of handling molecules, we just added to moleculARweb a new WebXR-based tool ('Build your own webAR views') where users can load 3D objects exported from $\mathrm{VMD}^{[18]}$ in Wavefront format (such as molecular representations, orbital density isosurfaces, X-ray diffraction meshes, etc.) and visualize them either on our regular webAR apps or in high-end webVR-compatible devices in which the molecules can be manipulated right away with bare hands ${ }^{[2]}$ (note that since these models are loaded as full objects, for the moment the 3D views are static). Importantly, the types of VR devices required for this are becoming more affordable and are increasingly adopting the WebXR standard, facilitating content development and device cross-compatibility. ${ }^{[10]}$

\subsection{More accurate, yet fast, physics and chemistry}

The other key point that needs development is the simulation of molecular mechanics, trying to improve realism yet keeping it simple and fast as in the rigid body implementation we used. The 'dream tool' should be able to produce realistic potential energy surfaces over conformations and also over bond formation and breaking. It is important to bear in mind that since in these tools the user controls what events happen and through which reaction coordinates, then aiming at considering entropy and full free energy landscapes is pointless - that's why we stress potential energy surfaces. Considering this, we propose that machine learning-based methods trained on high-quality data hold the key. We are in fact experimenting with interfacing the VMKs to ANI, a neural network trained from DFT data to compute potential energies for small molecules from their atomic coordinates within milliseconds. ${ }^{[19,20]}$ Although ANI-derived forces could in theory be used to drive dynamics, in practice this turns out too slow for our intended level of interactivity. Besides, in its current version ANI would only be suitable for a small subset of the applications discussed and limited to conformation-related studies on single molecules. However, as ANI and other methods evolve, we are confident that full physics, possibly even including reactivity, could become possible -the dream tool for education and possibly even for research in chemistry.

\section{Acknowledgements}

We acknowledge Paul Schalbetter (SV-IT, EPFL, Switzerland) for help setting up the website; Adrián Roitberg and Ignacio Pickering (U. Florida, USA) for help with PyTorch-ANI; Celeste Aguirre-Pranzoni (U. San Luis, Argentina) and numerous other chemistry teachers around the world for help and ideas on pedagogic material; T. Limpanuparb (Mahidol U., Thailand) for providing example molecules and Thai trans- lation; and Noah Burger (Kantonsschule Romanshorn, Switzerland) and Philipp Schumann (Kollegium Spiritus Sanctus Brig, Switzerland) for help preparing molecules for the ready-to-use set of molecules.

This work was supported by the Swiss National Foundation through grants Spark CRSK-1_190376 and Agora CRARP2_202370 to LAA, and by EPFL to MDP.

Received: December 20, 2021

[1] F. Cortés Rodríguez, G. Frattini, L. F. Krapp, H. Martinez-Hung, D. M. Moreno, M. Roldán, J. Salomón, L. Stemkoski, S. Traeger, M. Dal Peraro, J. Chem. Educ. 2021, 98, 2243, https://doi.org/10.1021/acs.jchemed.1c00179.

[2] F. Cortes Rodriguez, F, M. Dal Peraro, L. A. Abriata, ChemRxiv 2021.

[3] L. A. Abriata, DNA Cell Biol. 2021, https://doi.org/10.1089/dna.2021.0497.

[4] H. S. Fernandes, N. M. Cerqueira, S. F. Sousa, J. Chem. Ed. 2021, 98, 1789, https://doi.org/10.1021/acs.jchemed.0c01317.

[5] J. M. Argüello, R. E. Dempski, J. Chem. Ed. 2020, 97, 2327, https://doi.org/10.1021/acs.jchemed.0c00323.

[6] H. S. Gan, N. Y. K. Tee, M. R. Bin Mamtaz, K. Xiao, B. H.-P. Cheong, O. W. Liew, T. W. Ng, Biochem. Mol. Biol. Educ. 2018, 46, 245, https://doi.org/10.1002/bmb.21117.

[7] K. N. Plunkett, J. Chem. Educ. 2019, 96, 2628 , https://doi.org/10.1021/acs.jchemed.9b00607.

[8] L. A. Abriata, in 'Informatics', Vol. 4, Multidisciplinary Digital Publishing Institute, 2017, p. 28

[9] L. A. Abriata, Peer J Comput. Sci. 2020, 6, e260, https://doi.org/10.7717/peerj-cs.260.

[10] F. Cortés Rodríguez, M. Dal Peraro, L. A. Abriata, Nat. Comput. Sci. 2021, 1, 631, https://doi.org/10.1038/s43588-021-00142-8.

[11] L. A. Abriata, J. P. G. L. M. Rodrigues, M. Salathé, L. Patiny, Trends Biotechnol. 2018, 36, 473, https://doi.org/10.1016/j.tibtech.2017.11.009.

[12] C. Lacoursière, in 'The 48th Scandinavian Conference on Simulation and Modeling (SIMS 2007), 30-31 October, 2007, Göteborg (Särö)', Linköping University Electronic Press, 2007, pp. 40.

[13] B. Bienfait, P. Ertl, J. Cheminformatics 2013, 5, 24, https://doi.org/10.1186/1758-2946-5-24.

[14] M. Sitzmann, I. V. Filippov, M. C. Nicklaus, SAR QSAR Environ. Res. 2008, 19, 1, https://doi.org/10.1080/10629360701843540.

[15] M. Matsumoto, S. Saito, I. Ohmine, Nature 2002, 416, 409, https://doi.org/10.1038/416409a.

[16] L. Martinez, R. Andrade, E. G. Birgin, J. M. Martinez, J Comput. Chem. 2009, 30, 2157, https://doi.org/10.1002/jcc.21224.

[17] F. Cortés Rodriguez, C. Aguirre-Pranzoni, A. Príncipe, L. Abriata, Quimica Viva 2021, 20.

[18] W. Humphrey, A. Dalke, K. Schulten, J. Mol. Graph. 1996, 14, 33, https://doi.org/10.1016/0263-7855(96)00018-5.

[19] J. S. Smith, O. Isayev, A. E. Roitberg, Chem. Sci. 2017, 8, 3192 https://doi.org/10.1039/C6SC05720A.

[20] X. Gao, F. Ramezanghorbani, O. Isayev, J. Smith, A. Roitberg, J. Chem. Inf Model. 2020, 60, 3408, https://doi.org/acs.jcim.0c00451.

\section{License and Terms}

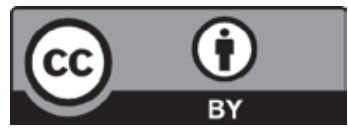

This is an Open Access article under the terms of the Creative Commons Attribution License CC BY 4.0. The material may not be used for commercial purposes.

The license is subject to the CHIMIA terms and conditions: (https://chimia.ch/chimia/about).

The definitive version of this article is the electronic one that can be found at https://doi.org/10.2533/chimia.2022. 